\title{
Regioselective Suzuki coupling on pyridinium $N$-(3,5-dibromoheteroar-2-yl)aminides
}

\author{
M. José Reyes, Rafael Castillo, M. Luisa Izquierdo and Julio Alvarez-Builla* \\ Departamento de Química Orgánica, Universidad de Alcalá, 28871 Alcalá de Henares, Madrid, Spain
}

Received 27 April 2006; revised 19 June 2006; accepted 20 June 2006

\begin{abstract}
A regioselective Suzuki-Miyaura cross-coupling reaction on $3^{\prime}, 5^{\prime}$-dibromo pyridinium $N$ - $\left(2^{\prime}\right.$-azinyl)aminides is reported. A series of $3^{\prime}$-aryl(or heteroaryl)-5'-bromo-pyridinium $N$ - $\left(2^{\prime}\right.$-pirazinyl)aminides were obtained in good yields. Two isomeric $3^{\prime}, 5^{\prime}$ diaryl pyridinium $N$-(2'-azinyl)aminides were also prepared.

(C) 2006 Elsevier Ltd. All rights reserved.
\end{abstract}

Functionalization of heterocycles through SuzukiMiyaura palladium-catalyzed cross-coupling has been established as a standard method for the synthesis of biaryl and heterobiaryl systems. ${ }^{1}$ Substituted pyridines and pyrazines are valuable intermediates in the pharmaceutical and flavour fields, so development of regioselective processes over both systems has an indubitable interest. In the past years, our research programme has been related with the synthetic utility of pyridinium $N$-(2'-azinyl)aminides $\mathbf{1}^{2}$ (Fig. 1) as building blocks in the synthesis of azine derivatives, ${ }^{3}$ and we recently reported preliminary results obtained in the crosscoupling reaction between arylboronic acids and pyridinium $N$-(5'-bromoheteroar-2'-yl)aminides $1 \mathbf{a}-\mathbf{c}^{4}$ (Fig. 1).

The promising results obtained from ylides 1a,b justified broadening of the process over other aminides, such as $N$-(3',5'-dibromopyrid-2'-yl)pyridinium aminide $1 \mathbf{1 c}^{3 \mathrm{c}}$ On this substrate, a double Suzuki reaction was performed, and different 3,5-disubstituted azines were obtained. ${ }^{4}$
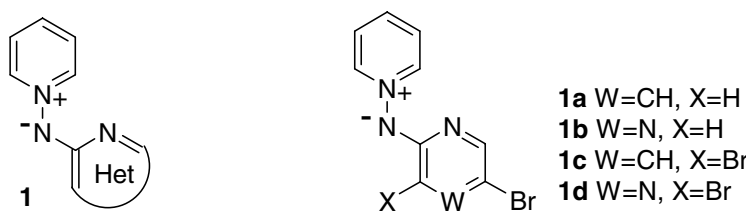

Figure 1.

\footnotetext{
*Corresponding author. Fax: +34 9188546 86; e-mail: julio. alvarez@uah.es
}

The same double coupling methodology on 1c, employing 3-pyridylboronic acid (Scheme 1), produced a low yield $(18 \%)$ of the expected bis-heteroaryl aminide $\mathbf{2 a}$, while the monosubstituted ylide $\mathbf{3 a}$ appeared as the main reaction product $(48 \%)$, in addition to $28 \%$ of starting material 1c that was recovered. Neither the use of a large excess of 3-pyridylboronic acid and/or palladium catalyst, nor longer reaction times, improved the yields of compound 2a.

The structure elucidation of 3a was solved by comparison of its ${ }^{1} \mathrm{H}$ NMR spectra with those of related compounds. $\mathrm{H}^{\prime}$ of the 2-aminopyridine ring appears more shielded when a bromine atom is in position $5^{\prime}-$, than when an arene is placed in the same position. ${ }^{3 b, 4}$ The following data were obtained for $\delta \mathrm{H}^{\prime}\left(\mathrm{CD}_{3} \mathrm{OD}\right)$ in compounds 1c, 2a and 3a: $7.74 \mathrm{ppm}, 8.03 \mathrm{ppm}$ and $7.71 \mathrm{ppm}$, respectively. This result pointed out a considerable degree of regioselectivity in the Suzuki process, when $\quad N-\left(3^{\prime}, 5^{\prime}\right.$-dibromo-2'-pyridin-2'-yl)pyridinium aminide 1c was reacted with pyridyl-3-boronic acid.

Regioselectivity in palladium cross-coupling reactions of di-haloazines, having two identical halogen atoms in different positions of the ring, has not been often described in the literature. ${ }^{\text {5a }}$ On uncharged systems, 2,5-dibromopyridine undergoes a regioselective palladium-catalyzed coupling with terminal acetylenes and arylzinc halides, ${ }^{5 b}$ and 2,5-dichloropyridine selectively reacts at 2-position with phenylboronic acid. ${ }^{5 \mathrm{c}, \mathrm{d}}$ Moreover, the synthesis of 2-aryl-6-chloronicotinamides via Suzuki coupling of 2,6-dichloronicotinamide with aryl boronic acids has also been described, and in this case, regioselectivity 


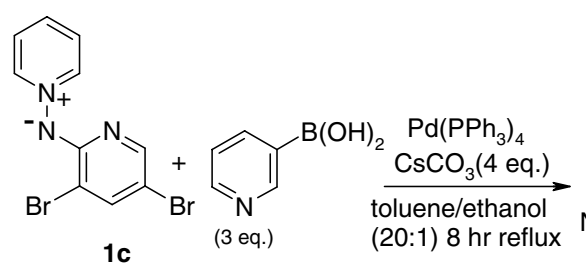

Scheme 1.

was achieved by chelation of the palladium( 0 ) species by the amide group. ${ }^{5 \mathrm{e}}$ Furthermore, Thompson et al. obtained the 3,5-diheteroaryl product by Suzuki coupling of 3,5-dibromopyridin-2-ylamine and 2-methoxypyridin-3-yl boronic acid, but nothing appears in the letter concerning the presence of monocoupled products in the mixture. ${ }^{5 f}$ Regioselective Suzuki coupling has also been reported in certain dihaloazine and -diazine systems, but only when the reactivity differences between the two halogens was well defined. ${ }^{6}$

The above cited results encouraged us to perform new experiments to test the regioselectivity of the Suzuki reaction of $N$-( $3^{\prime}, 5^{\prime}$-dibromopyrid-2'-yl)pyridinium aminide $1 c$ and $N$-( $3^{\prime}, 5^{\prime}$-dibromopyrazin-2 - -yl)pyridinium aminide 1d. Table 1 shows the results obtained for parallel coupling tests on 1c and 1d with three different boronic acids, in the presence of $\mathrm{Pd}\left(\mathrm{PPh}_{3}\right)_{4}(5 \%)$, under slightly different conditions (A and B) (Scheme 2). In all cases the reaction mixtures, after $5 \mathrm{~h}$ reflux, were analyzed by HPLC/MS. ${ }^{7}$

The two aryl isomers $\mathbf{3}$ and $\mathbf{4}$ were detected together with the corresponding 3,5-disubstituted aminide $\mathbf{2}$ and a small quantity of starting material $\mathbf{1}$. In all cases, a preference for substitution on position $3^{\prime}$ - was observed (Scheme 2).

From these data, it appears that both the nature of the aminide substrate and the boronic acid used are the main factors influencing the degree of selectivity obtained. Aminide 1d and, in this case, method B produced the best results with phenyl boronic acid, with a higher yield and regioselectivity observed (Table 1, entries 5 and 6).

Although a model explaining the selectivity observed in mono coupling reaction towards one of the bromine groups in aminides $\mathbf{1}$ is not yet fully available, one important factor should be the increased stability of the palladium intermediate 5 (Fig. 2) with respect to its opposite regioisomer. However, comparing results between $\mathbf{1 c}$ and $\mathbf{1 d}$, the regioselectivity is clearly higher in the pyrazine derivative, so the second ring nitrogen should have an additional influence. In a recent paper, Yang and Jiang ${ }^{8}$ reported a similar selectivity for the Suzuki coupling on 2,5-dibromo-3-methoxy pyrazine, a non-charged substrate, which with one equivalent of phenylboronic acid, yielded the 2-phenyl derivative in a high yield.

In Table 2, the results obtained for the Suzuki coupling of $N$-( $3^{\prime}, 5^{\prime}$-dibromopyrazin-2'-yl)pyridinium aminide 1d with aryl and heteroarylboronic acids, following method $\mathrm{B}$ are presented. In all cases a high degree of regioselectivity for the 3-position was observed, with both aryl and heteroarylboronic acids, and pyridinium $N-\left(3^{\prime}\right.$-aryl (or heteroaryl)-5'-bromopyrazin-2'-yl)aminides 3 were obtained in good yields. ${ }^{9}$

The availability of aminides $\mathbf{3}$ allowed the synthesis of two isomeric $3^{\prime}, 5^{\prime}$-disubstituted $N$-pyrazin-2'-ylpyridinium aminides $\mathbf{6 d}, \mathbf{h}$ which were obtained when an additional Suzuki coupling was achieved on ylides $\mathbf{3 d}$ and $\mathbf{3 h}$,

Table 1. Suzuki coupling of aminides $\mathbf{1}, \mathbf{d}$ and $\mathrm{R}-\mathrm{B}(\mathrm{OH})_{2}$ : a regioselectivity study

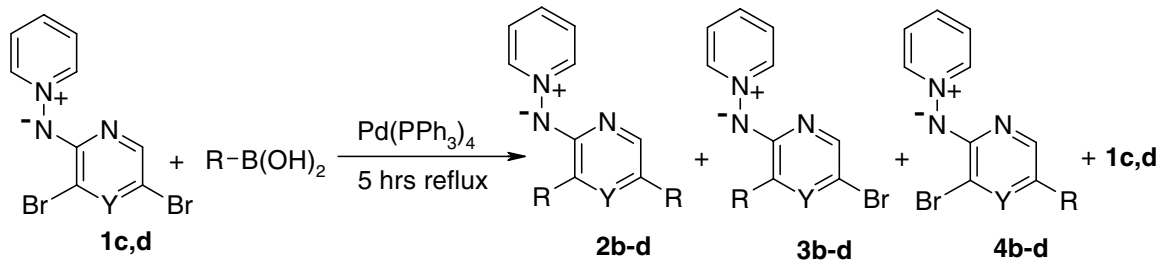

\begin{tabular}{|c|c|c|c|c|c|c|c|c|}
\hline Entry & Aminide & $\mathrm{Y}$ & $\mathrm{R}$ & Method $^{\mathrm{a}}$ & $2 \mathbf{b}-\mathbf{d}^{\mathrm{b}}$ Yield $(\%)$ & 3b- $\mathbf{d}^{\mathrm{b}}$ Yield $(\%)$ & 4b- $\mathbf{d}^{\mathrm{b}}$ Yield $(\%)$ & $\mathbf{1 c}, \mathbf{d}^{\mathrm{b}}$ Yield $(\%)$ \\
\hline 1 & $1 \mathrm{c}$ & $\mathrm{CH}$ & $\mathrm{Ph}$ & $\mathrm{A}$ & 14 & 52 & 16 & 18 \\
\hline 2 & $1 c$ & $\mathrm{CH}$ & $\mathrm{Ph}$ & $\mathrm{B}$ & 10 & 36 & 23 & 31 \\
\hline 3 & $1 \mathrm{c}$ & $\mathrm{CH}$ & $p$-Tol. & A & 22 & 36 & 27 & 15 \\
\hline 4 & $1 \mathrm{c}$ & $\mathrm{CH}$ & $p$-Tol. & $\mathrm{B}$ & 29 & 32 & 30 & 10 \\
\hline 5 & 1d & $\mathrm{N}$ & $\mathrm{Ph}$ & $\mathrm{A}$ & 8 & 61 & 26 & 5 \\
\hline 6 & 1d & $\mathrm{N}$ & $\mathrm{Ph}$ & $\mathrm{B}$ & 25 & 67 & 3 & 5 \\
\hline
\end{tabular}

${ }^{\mathrm{a}}$ Method A: $\mathrm{Cs}_{2} \mathrm{CO}_{3}$ (4 equiv) as a base in toluene:ethanol (20:1). Method B: $\mathrm{K}_{2} \mathrm{CO}_{3}$ (10 equiv) as a base in toluene:ethanol (4:1).

${ }^{\mathrm{b}}$ Yields determined by HPLC/MS. ${ }^{7}$ 
<smiles>c1cc[nH+]cc1</smiles>

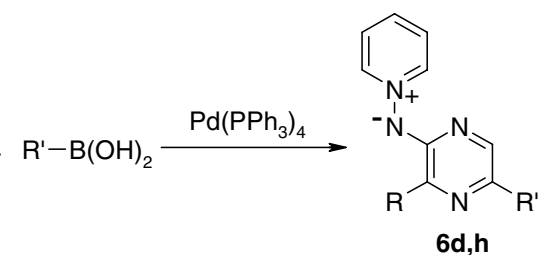

3d,h

\begin{tabular}{lcrc} 
Compd. & R & R $^{\prime}$ & Yield(\%) \\
\hline 6d & Phenyl & 3-Pyridyl & 53 \\
6h & 3-Pyridyl & Phenyl & 68
\end{tabular}

Scheme 2.

employing 4-pyridyl- and phenylboronic acids, respectively ${ }^{10}$ (Scheme 2). In this way, the method provides a straightforward strategy to obtain unsymmetrical $3^{\prime}, 5^{\prime}$ disubstituted aminides $\mathbf{6}$, and not only the symmetrical derivatives as has been described earlier. ${ }^{4}$<smiles>C[Pb](C)(C)[N+](c1ncc(Br)cc1I)[n+]1ccccc1</smiles>

Figure 2.

In conclusion, a regioselective Suzuki-Miyaura crosscoupling process has been observed, on pyridinium $N$-(3',5'-dibromopirazyn-2'-yl)aminides similar to $\mathbf{1 d}$. Although less evident, the phenomenon appears also in their pyridine analogue. As a general methodology, the process can be useful to selectively functionalize the 3position of the pyrazine ring. Two successive cross-coupling reactions over 1d gave satisfactory yields in aminides 6 with a non-symmetrical substitution (Scheme 2)

Table 2. Regioselective $\mathrm{C}-\mathrm{C}$ coupling of aminide $\mathbf{1 d}$ and boronic acids

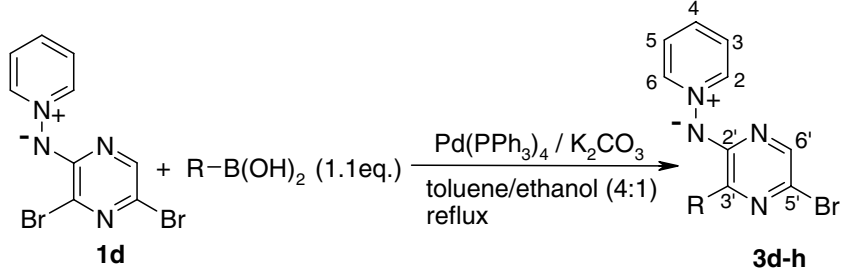

\begin{tabular}{|c|c|c|c|c|c|}
\hline Entry & Product & $\mathrm{R}$ & Reaction time (h) & $\mathrm{Mp}\left({ }^{\circ} \mathrm{C}\right)$ & Yield $(\%)^{\mathrm{a}}$ \\
\hline 1 & $3 d$ & & 3 & 198-199 & 75 \\
\hline 2 & $3 e$ & & 4 & $200-202$ & 50 \\
\hline 3 & $3 f$ & & 5 & $182-183$ & 71 \\
\hline 4 & $3 g$ & & 1 & $190-192$ & 65 \\
\hline 5 & $3 \mathrm{~h}$ & & 2 & $170-172$ & 65 \\
\hline 6 & $3 \mathbf{i}$ & & 4 & $229-231$ & 77 \\
\hline 7 & $3 \mathbf{j}$ & & 16 & $236-238$ & 60 \\
\hline 8 & $3 \mathbf{k}$ & & 12 & $163-165$ & 84 \\
\hline 9 & 31 & & 12 & 206-208 & 81 \\
\hline
\end{tabular}

${ }^{\text {a }}$ Isolated yield. Method B, as described in Table 1, was used. 
that can undergo, as we have described earlier, ${ }^{3 a, c}$ either an $\mathrm{N}-\mathrm{N}$ reduction to produce 3,5-disubstituted 2-aminopyrazines $^{3 \mathrm{a}, \mathrm{c}}$ or a regioselective alkylation process at the exocyclic nitrogen followed by a similar $\mathrm{N}-\mathrm{N}$ reduction, to yield 3,5-disubstituted $N$-alkyl-2-aminopyrazines. ${ }^{3 \mathrm{~d}, \mathrm{f}, \mathrm{g}}$ So, the whole approach might give access to useful pyrazine intermediates. Efforts in this direction are in progress in our laboratory.

\section{Acknowledgements}

The authors wish to thank the Comisión Interministerial de Ciencia y Tecnología (CICYT-BQU2001-1508) and the Universidad de Alcalá (UAH GC2005/006) for financial support, and the Ministerio de Educación y Cultura (MEC) for two studentships (M.J.R. and R.C.).

\section{References and notes}

1. (a) Miyaura, N.; Suzuki, A. Chem. Rev. 1995, 95, 24572495; (b) Suzuki, A. J. Organomet. Chem. 1999, 576, 147168; (c) Kotha, S.; Lahiri, L.; Kashinath, D. Tetrahedron 2002, 58, 9633-9695; (d) Agrofoglio, L. A.; Gillaizeau, I.; Saito, Y. Chem. Rev. 2003, 103, 1875-1916; (e) Li, J. J.; Gribble, G. W. Palladium in Heterocyclic Chemistry; Pergamon, 2000.

2. (a) Ollis, D. W.; Stanforth, S. P. Tetrahedron 1985, 41, 2239-2339; (b) Sliva, W. Heterocycles 1991, 32, 22412272; (c) Vaquero, J. J.; Alvarez-Builla, J. Adv. Nitrogen Heterocycl. 2000, 4, 159-250.

3. (a) Carceller, R.; García-Navío, J. L.; Izquierdo, M. L.; Alvarez-Builla, J. Tetrahedron Lett. 1993, 34, 2019-2020; (b) Carceller, R.; García-Navío, J. L.; Izquierdo, M. L.; Alvarez-Builla, J.; Fajardo, M.; Gómez-Sal, P.; Gago, F. Tetrahedron 1994, 50, 4995-5012; (c) Burgos, C.; Delgado, F.; García-Navío, J. L.; Izquierdo, M. L.; Álvarez-Builla, J. Tetrahedron 1995, 31, 8649-8654; (d) García de Viedma, A.; Martinez-Barrasa, V.; Burgos, C.; Izquierdo, M. L.; Álvarez-Builla, J. J. Org. Chem. 1999, 64, 1007-1010; (e) De la Rosa, R.; Martinez-Barrasa, V.; Burgos, C.; Alvarez-Builla, J. Tetrahedron Lett. 2000, 41, 5837-5840; (f) Martínez-Barrasa, V.; Delgado, F.; Burgos, C.; GarcíaNavío, J. L.; Izquierdo, M. L.; Álvarez-Builla, J. Tetrahedron 2000, 56, 2481-2490; (g) Reyes, M. J.; Delgado, F.; Izquierdo, M. L.; Alvarez-Builla, J. Tetrahedron 2002, 58, 8573-8579; (h) Nuñez, A.; García de Viedma, A.; Martínez-Barrasa, V.; Burgos, C.; Alvarez-Builla, J. Synlett 2002, 7, 1093-1096; (i) Sánchez, A.; Nuñez, A.; AlvarezBuilla, J.; Burgos, C. Tetrahedron 2004, 60, 11843-11850.

4. Reyes, M. J.; Izquierdo, M. L.; Alvarez-Builla, J. Tetrahedron Lett. 2004, 45, 8713-8715.

5. (a) Stanforth, S. P. Tetrahedron 1998, 54, 263-303; (b) Tilley, J. W.; Zawoiski, S. J. Org. Chem. 1988, 53, 386390; (c) Mitchell, M. B.; Wallbank, P. J. Tetrahedron Lett. 1991, 32, 2273-2276; (d) Naji, M. A.; McKillop, A.; Mitchell, M. B.; Rebelo, R. A.; Wllbank, P. J. Tetrahedron 1992, 48, 8117-8126; (e) Yang, W.; Wang, Y.; Corte, J. R. Org. Lett. 2003, 5, 3131-3134; (f) Thompson, A. E.; Hughes, G.; Batsanov, A. S.; Bryce, M. R.; Parry, P. R.; Tarbit, B. J. Org. Chem. 2005, 70, 388-390.
6. (a) Gong, Y.; Pauls, H. W. Synlett 2000, 6, 829-831; (b) Sotelo, E.; Raviña, E. Synlett 2002, 2, 223-226; (c) Bouillon, A.; Voisin, A. S.; Robie, A.; Lancelot, J.-C.; Rault, S. J. Org. Chem. 2003, 68, 10178-10180; (d) Gong, Y.; He, W. Heterocycles 2004, 62, 851-856.

7. HPLC conditions: Column, Luna C-18 $(150 \times 4.6 \mathrm{~mm})$ from Jasco Analitica; Eluent, $\mathrm{HCOOH}\left(4 \%\right.$ in $\left.\mathrm{H}_{2} \mathrm{O}\right)(\mathrm{A})$, $\mathrm{H}_{2} \mathrm{O}(\mathrm{B})$ and $\mathrm{CH}_{3} \mathrm{CN}(\mathrm{C})$; Gradient, $93 \%$ B-4.5\% B in $25 \mathrm{~min}, \mathrm{~A}=2.5 \% t=0-25 \mathrm{~min}$; Flow, $1.0 \mathrm{~mL} / \mathrm{min} ; \lambda, 280$, 284 and $210 \mathrm{~nm}$. Experiments were performed on an Agilent 1100 HPLC/MS.

8. Yang, C.-G.; Jiang, B. J. Org. Chem. 2002, 67, 9392-9396.

9. General procedure for monocoupling: $\mathrm{Pd}\left(\mathrm{PPh}_{3}\right)_{4}(57 \mathrm{mg}$, $5 \% \mathrm{mmol})$, boronic acid $(1.1 \mathrm{mmol})$, aminide $1 \mathrm{~d}(330 \mathrm{mg}$, $1 \mathrm{mmol})$ and $\mathrm{K}_{2} \mathrm{CO}_{3}(10 \mathrm{mmol})$ were stirred under argon and refluxed in a mixture of toluene:ethanol 4:1 (15 mL) until no starting material was detected by TLC. The mixture was filtered through celite and washed with acetonitrile. The filtrates were combined and evaporated to dryness. The residue was purified by flash chromatography on a silica gel column using ethanol as eluent, and recrystallized from the appropriate solvent.

$N$ - $\left(5^{\prime}\right.$-Bromo- $3^{\prime}$-phenylpyraz-2'-yl)pyridinium aminide (3d): Orange solid (245 mg, 75\%), mp 198-199 ${ }^{\circ} \mathrm{C}$ (AcOEt), IR $(\mathrm{KBr}) v_{\max }\left(\mathrm{cm}^{-1}\right): 1484,1404,1372,1239,696 \mathrm{~cm}^{-1} ;{ }^{1} \mathrm{H}$ NMR (300 MHz, $\left.\mathrm{CD}_{3} \mathrm{OD}\right): \delta$ (ppm) $8.71(2 \mathrm{H}, \mathrm{dd}, J=7.0$ and $1.3 \mathrm{~Hz}, H 2(6)) ; 8.15(1 \mathrm{H}, \mathrm{tt}, J=7.6$ and $1.3 \mathrm{~Hz}, H 4)$; $8.13\left(2 \mathrm{H}, \mathrm{dd}, J=8.4\right.$ and $\left.1.5 \mathrm{~Hz}, H 2^{\prime \prime}\left(6^{\prime \prime}\right)\right) ; 7.87(2 \mathrm{H}$, dd, $J=7.6$ and $7.0 \mathrm{~Hz}, H 3(5)) ; 7.58\left(1 \mathrm{H}, \mathrm{s}, H 6^{\prime}\right) ; 7.44(3 \mathrm{H}, \mathrm{m}$, $H 3^{\prime \prime}\left(5^{\prime \prime}\right)$ and $\left.H 4^{\prime \prime}\right) ;{ }^{13} \mathrm{C}$ NMR $\left(75 \mathrm{MHz}, \mathrm{CD}_{3} \mathrm{OD}\right): \delta 156.4$ $\left(C 2^{\prime}\right), 142.3(C 2(6)), 141.9\left(C 3^{\prime}\right), 140.0\left(C 6^{\prime}\right), 137.3\left(C 1^{\prime \prime}\right)$, $134.1(C 4), 129.1\left(C 2^{\prime \prime}\left(6^{\prime \prime}\right)\right), 128.4\left(C 4^{\prime \prime}\right), 127.7\left(C 3^{\prime \prime}\left(5^{\prime \prime}\right)\right)$, $126.0(C 3(5)), 121.7\left(C 5^{\prime}\right)$. Anal. Calcd for $\mathrm{C}_{15} \mathrm{H}_{11} \mathrm{BrN}_{4}$ : C, 55.07; H, 3.39; N, 17.12. Found: C, 55.28; H, 3.60; N, 16.93.

10. General procedure for compounds 6: $\mathrm{Pd}\left(\mathrm{PPh}_{3}\right)_{4}(57 \mathrm{mg}$, $5 \% \mathrm{mmol})$, boronic acid $(1.5 \mathrm{mmol})$, aminide $3(1 \mathrm{mmol})$ and $\mathrm{K}_{2} \mathrm{CO}_{3}(10 \mathrm{mmol})$ were stirred and refluxed under argon, in a mixture of toluene:ethanol 4:1 (15 mL) until no starting material was detected by TLC. The mixture was filtered through celite and washed with acetonitrile. The filtrates were combined and evaporated to dryness. The residue was purified by flash chromatography on a silica gel column using ethanol as eluent, and recrystallized from the appropriate solvent.

$N$ - $\left[3^{\prime}-\right.$ Phenyl-5'-( $3^{\prime \prime}-$ pyridyl $)$ pyraz-2'-yl)pyridinium aminide (6d): Orange solid $(173 \mathrm{mg}, 53 \%)$, mp $173-176{ }^{\circ} \mathrm{C}(\mathrm{EtOH}-$ AcOEt), IR (KBr) $v_{\max }\left(\mathrm{cm}^{-1}\right): 1554,1501,1468,1401$, $1368,1303,1252,1142,799,754,703,664 \mathrm{~cm}^{-1} ;{ }^{1} \mathrm{H}$ NMR (300 MHz, CD $3 \mathrm{OD}): \delta(\mathrm{ppm}) 9.06(1 \mathrm{H}, \mathrm{d}, J=2.2 \mathrm{~Hz}$, $\left.H 2^{\prime \prime \prime}\right) ; 8.71(2 \mathrm{H}, \mathrm{dd}, J=6.8$ y $1.3 \mathrm{~Hz}, H 2(6)) ; 8.39(1 \mathrm{H}, \mathrm{dd}$, $J=4.8$ and $\left.1.7 \mathrm{~Hz}, H 6^{\prime \prime \prime}\right) ; 8.30(1 \mathrm{H}$, ddd, $J=8.1,2.2$ and $\left.1.7 \mathrm{~Hz}, H 4^{\prime \prime \prime}\right) ; 8.26(2 \mathrm{H}, \mathrm{dd}, J=8.4$ and $1.6 \mathrm{~Hz}$, $\left.H 2^{\prime \prime}\left(6^{\prime \prime}\right)\right) ; 8.13(1 \mathrm{H}, \mathrm{tt}, J=7.5$ y $1.3 \mathrm{~Hz}, H 4) ; 8.06(1 \mathrm{H}, \mathrm{s}$, $\left.H 6^{\prime}\right) ; 7.84(2 \mathrm{H}, \mathrm{dd}, J=7.5$ and $6.8 \mathrm{~Hz}, H 3(5)) ; 7.44(4 \mathrm{H}$, $\mathrm{m}, H 3^{\prime \prime}\left(5^{\prime \prime}\right), H 4^{\prime \prime}$ and $\left.H 5^{\prime \prime \prime}\right) ;{ }^{13} \mathrm{C}$ NMR (75 MHz. $\left.\mathrm{CD}_{3} \mathrm{OD}\right)$ : $\delta 159.3\left(C 2^{\prime}\right), 147.4\left(C 6^{\prime \prime \prime}\right), 146.0\left(C 2^{\prime \prime \prime}\right), 145.6(C 2(6))$, $142.4\left(C 3^{\prime}\right), 139.7\left(C 1^{\prime \prime}\right), 139.4(C 4), 137.5\left(C 6^{\prime}\right), 135.6$ $\left(C 3^{\prime \prime \prime}\right.$ or $\left.C 5^{\prime}\right), 134.8\left(C 3^{\prime \prime \prime}\right.$ or $\left.C 5^{\prime}\right), 133.5\left(C 4^{\prime \prime \prime}\right), 130.2$ $\left(C 2^{\prime \prime}\left(6^{\prime \prime}\right)\right), 129.3\left(C 4^{\prime \prime}\right), 128.7\left(C 3^{\prime \prime}\left(5^{\prime \prime}\right)\right), 128.5(C 3(5))$, $125.1\left(C 5^{\prime \prime \prime}\right)$. Anal. Calcd for $\mathrm{C}_{20} \mathrm{H}_{15} \mathrm{~N}_{5} \cdot 1 / 2 \mathrm{H}_{2} \mathrm{O}$ : C, 71.84; H, 4.82; N, 20.94. Found: C, 71.80; H, 4.82; N, 20.63. MS (CI, m/z): 326 (57, M+1), 277 (20), 249 (100), 80 (43). 